\title{
A Model for Ternary Projective Relations between Regions
}

\author{
Roland Billen ${ }^{1}$ and Eliseo Clementini ${ }^{2}$ \\ ${ }^{1}$ Dept. of Geography and Geomatics \\ University of Glasgow \\ Glasgow G12 8QQ, Scotland, UK \\ rbillen@geog.gla.ac.uk \\ ${ }^{2}$ Dept. of Electrical Engineering \\ University of L'Aquila \\ I-67040 Poggio di Roio (AQ), Italy \\ eliseo@ing.univaq.it
}

\begin{abstract}
Current spatial database systems offer limited querying capabilities beyond topological relations. This paper introduces a model for projective relations between regions to support other qualitative spatial queries. The relations are ternary because they are based on the collinearity invariant of three points under projective geometry. The model is built on a partition of the plane in five regions that are obtained from projective properties of two reference objects: then, by considering the empty/non empty intersections of a primary object with these five regions, the model is able to distinguish between 31 different projective relations.
\end{abstract}

\section{Introduction}

A formal geometric definition of spatial relations is needed to build reasoning systems on them and facilitate a standard implementation in spatial database systems. This is what happens for some models of topological relations, like the 9-intersection [6] and the calculus-based method - CBM [3]. These models provide formal definitions for the relations, establish reasoning mechanisms to find new relations from a set of given ones [5] and, as part of the OpenGIS specifications [17] and ISO/TC 211 standard, have been implemented in several commercial geographic information systems (GISs) and spatial database systems.

Topological relations take into account an important part of geometric knowledge and can be used to formulate qualitative queries about the connection properties of close spatial objects, like "retrieve the lakes that are inside Scotland". Other qualitative queries that involve disjoint objects cannot be formulated in topological terms, for example: "the cities that are between Glasgow and Edinburgh", "the lakes that are surrounded by the mountains", "the shops that are on the right of the road", the building that is before the crossroad". All these examples can be seen as semantic interpretations of underlying projective properties of spatial objects. As discussed in [1], geometric properties can be subdivided in three groups: topological, projective and metric. Most qualitative relations between spatial objects can be defined in terms 
of topological or projective properties [24], with the exception of qualitative distance and direction relations (such as close, far, east, north) that are a qualitative interpretation of metric distances and angles [2].

Invariants are geometric properties that do not change after a certain class of transformations: topological invariants are properties that are maintained after a topological transformation (a bicontinuous mapping or homeomorphism) and projective invariants are properties that are maintained after a projective transformation (projection). Likewise topological relations, which are defined by using the connectedness topological invariant, projective relations are defined by using the collinearity projective invariant, which is the property of three collinear points being still collinear after an arbitrary number of projections. A main difference in the treatment of topological relations and projective relations is that, while basic topological relations are binary, basic projective relations are ternary because they are defined on the collinearity of three objects. Still, we can identify some special cases of binary projective relations (e.g., an object is inside the convex hull of another). Other binary relations, such as surrounded by, can be derived as a consequence of the model of ternary relations. In the present paper, we will limit the treatment to the basic ternary relations, while unary projective operators and binary projective relations will be part of further developments of the model. To have a qualitative understanding of projective relations, it is sufficient to think to different twodimensional views of a three-dimensional real world scene of objects: changing the point of view, metric aspects such distances and angles among the objects appear to be different, but there are properties that are common in all the views. These common properties are projective properties.

In this paper, we propose a model for representing the projective relations between any three regions of the plane. One of these regions acts as the primary object and the other two as reference objects for the relation. We will show how by using only projective concepts it is possible to partition the plane in five regions with respect to the reference objects. Then, the model, called the 5-intersection, is able to differentiate between 31 different projective relations that are obtained by computing the intersection of the primary object with the five regions that are determined by the reference objects. Though in this paper we discuss the model for regions, the 5intersection can be applied to other spatial objects such as points and lines. Other developments not treated in this paper will be to establish a reasoning system with projective relations to infer unknown relations from a set of given relations: this will be based on the algebraic properties of projective relations.

In first approximation, this work can be compared to research on qualitative relations dealing with relative positioning or cardinal directions $[8,10,15,16,19,20]$ and also path relations [13]. Most approaches consider binary relations to which is associated a frame of reference [11]. But most of them, even when explicitly related to projective geometry, never avoid the use of metric properties (minimum bounding rectangles, angles, etc.) and external frames of reference (such as a grid). To this respect, the main difference in our approach is that we only deal with projective invariants, disregarding distances and angles. Most work on projective relations deals with point abstractions of spatial features and limited work has been devoted to extended objects $[10,14,23]$. In [4], the authors use spheres surrounding the objects to take into account the shape of objects in relative orientation. The projective relations that are introduced in our paper are influenced by the size and shape of the three objects involved in a relation. The acceptance areas of the relations are truly 
based on the projective properties of the objects. Early work on projective relations such as "between" was developed by [9]. Freksa's double-cross calculus [7] is similar to our approach in the case of points. Such a calculus, as it has been further discussed in $[12,21]$, is based on ternary directional relations between points. However, in Freksa's model, an intrinsic frame of reference centred in a given point partitions the plane in four quadrants that are given by the front-back and right-left dichotomies. This leads to a greater number of qualitative distinctions with different algebraic properties and composition tables.

The paper is organized as follows. We start in Section 2 with developing a model for ternary projective relations between points: this is a natural starting point because the collinearity invariant applies to three points. Such a model is a simplified version of the model for regions and is very useful to understand the plausibility of the relations. In Section 3, we introduce the 5-intersection model for ternary projective relations between regions, giving the definitions, the algebraic properties and examples of the geometric configurations. In Section 4, we draw short conclusions and discuss the future developments of this model.

\section{Projective Relations between Points}

Our basic set of projective relations is based on the most important geometric invariant in a projective space: the collinearity of three points. Therefore, the nature of projective relations is intrinsically ternary. It is not possible to speak about binary projective relations except in some special or derived cases, and also in this cases the third object cannot be said to be absent, but rather to be hidden. For example, an "object $A$ is in front of object $B$ ": this is linguistically a binary relation, but it implies that object $B$ has an intrinsic frame of reference [18] that determines which is its front and that takes the role of the 'third point'. Analogously, considering the proposition "point $P$ is to the right of line $L$ ", we have a derived binary relation because the direction of the line is defined by two points.

As a first step, we are going to define the basic ternary projective relations between points. Projective relations between points have a straightforward definition because they are related to common concepts of projective geometry [22]. The results of this section will be the basis for introducing the more complex definitions of projective relations between regions in Section 3.

\section{1 “Collinear" Relation}

The collinearity relation can be considered the most important relation from which all the others are obtained. The embedding space we are considering is $R^{2}$.

Definition 1. A point $P_{1}$ is collinear to two given points $P_{2}$ and $P_{3}$, collinear $\left(P_{1}, P_{2}, P_{3}\right)$, if one of the following conditions holds:
(a) $P_{2}=P_{3}$;
(b) $P_{1} \in \overline{P_{2} P_{3}}$. 


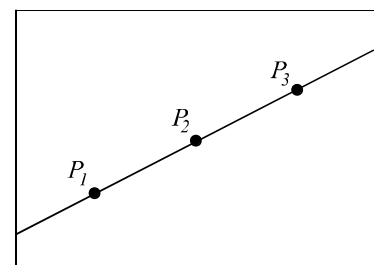

(a)

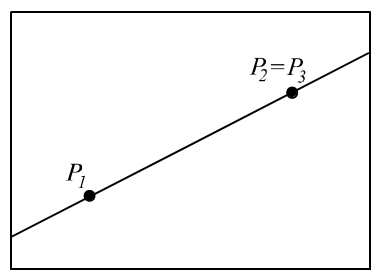

(b)

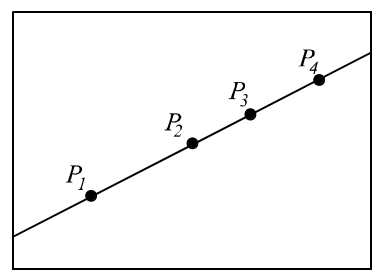

(c)

Fig. 1. The relation collinear between three points (a); the special case of coincidence of points $P_{2}$ and $P_{3}$ (b); illustration of the transitivity property (c)

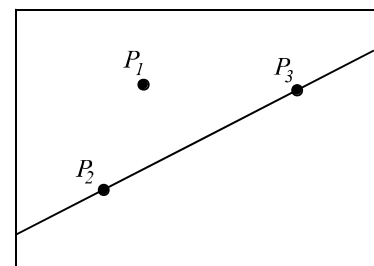

(a)

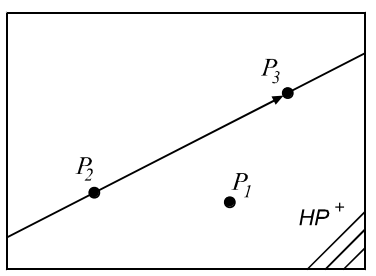

(b)

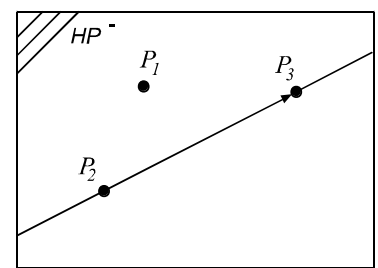

(c)

Fig. 2. The relation aside (a) and the relations rightside (b) and leftside (c)

Rephrasing Definition 1, the first point $P_{1}$ is collinear to two other points $P_{2}$ and $P_{3}$ if $P_{1}$ belongs to the line that passes through $P_{2}$ and $P_{3}$ (part (b)). The part (a) of the definition takes into account the trivial case where $P_{2}$ and $P_{3}$ are coincident and therefore they cannot define a line. Because there is always a line that passes through two points, we assume that the collinearity is also true in this case. The relation collinear is illustrated in Fig. 1(a-b).

Whenever we write the notation collinear $\left(P_{1}, P_{2}, P_{3}\right)$, the first of the three points corresponds to the one that holds the relation with the other two points and the other two points are defining a line. This order in the arguments of the relation is valid also for other ternary relations.

The properties of collinearity allow us to conclude that it is an equivalence relation. An equivalence relation is usually defined for binary relations by showing that they are reflexive, symmetric and transitive. An equivalence relation generates a partition in equivalence classes. With regard to the ternary collinearity relation, analogously we give below the properties that are divided in three groups (reflexivity, symmetry and transitivity). The equivalence classes that are generated are the classes of all collinear points, which are also all the lines of the space.

1. The collinear relation is reflexive. For all $P_{1}, P_{2} \in R^{2}$ :

a. collinear $\left(P_{1}, P_{1}, P_{1}\right)$;

b. collinear $\left(P_{1}, P_{2}, P_{2}\right)$;

c. collinear $\left(P_{1}, P_{1}, P_{2}\right)$;

d. collinear $\left(P_{1}, P_{2}, P_{1}\right)$. 
2. The collinear relation is symmetric. For all $P_{1}, P_{2}, P_{3} \in R^{2}$ :

a. collinear $\left(P_{1}, P_{2}, P_{3}\right) \Rightarrow$ collinear $\left(P_{1}, P_{3}, P_{2}\right)$;

b. collinear $\left(P_{1}, P_{2}, P_{3}\right) \Rightarrow$ collinear $\left(P_{2}, P_{1}, P_{3}\right)$;

c. collinear $\left(P_{1}, P_{2}, P_{3}\right) \Rightarrow$ collinear $\left(P_{3}, P_{1}, P_{2}\right)$.

3. The collinear relation is transitive (see Fig. 1(c)). For all $P_{1}, P_{2}, P_{3}, P_{4} \in R^{2}$ : $\operatorname{collinear}\left(P_{1}, P_{2}, P_{3}\right) \wedge$ collinear $\left(P_{2}, P_{3}, P_{4}\right) \Rightarrow \operatorname{collinear}\left(P_{1}, P_{3}, P_{4}\right)$.

\section{2 "Aside" Relation}

The aside relation is the complement of the collinear relation (see Fig. 2(a)).

Definition 2. A point $P_{1}$ is aside of two given points $P_{2}$ and $P_{3}$ aside $\left(P_{1}, P_{2}, P_{3}\right)$, if $P_{1} \notin{\overline{P_{2} P_{3}}}_{3}$ and $P_{2} \neq P_{3}$.

The aside relation could simply be defined as aside $\left(P_{1}, P_{2}, P_{3}\right) \Leftrightarrow \neg$ collinear $\left(P_{1}, P_{2}, P_{3}\right)$. The properties of the aside relation are restricted to the symmetry group:

1. The aside relation is symmetric.

$$
\begin{aligned}
& \operatorname{aside}\left(P_{1}, P_{2}, P_{3}\right) \Rightarrow \operatorname{aside}\left(P_{1}, P_{3}, P_{2}\right) \\
& \operatorname{aside}\left(P_{1}, P_{2}, P_{3}\right) \Rightarrow \operatorname{aside}\left(P_{2}, P_{1}, P_{3}\right) \\
& \operatorname{aside}\left(P_{1}, P_{2}, P_{3}\right) \Rightarrow \operatorname{aside}\left(P_{3}, P_{1}, P_{2}\right)
\end{aligned}
$$

\section{3 "Rightside" and "Leftside" Relations}

By considering the two halfplanes determined by the oriented line $\overrightarrow{P_{2} P_{3}}$, respectively the halfplane to the right of the line, which we indicate with $H^{+}\left(\overrightarrow{P_{2} P_{3}}\right)$, and the halfplane to the left of the line, which we indicate with $H P^{-}\left(\overrightarrow{P_{2} P_{3}}\right)$, we refine the configurations described by the relation aside $\left(P_{1}, P_{2}, P_{3}\right)$ in two distinct parts that are described by the relations rightside and leftside (see Fig. 2(b-c)).

Definition 3. A point $P_{1}$ is rightside of two given points $P_{2}$ and $P_{3}$, rightside $\left(P_{1}, P_{2}, P_{3}\right)$, if $P_{1} \in H P^{+}\left(\overrightarrow{P_{2} P_{3}}\right)$.

Definition 4. A point $P_{1}$ is leftside of two given points $P_{2}$ and $P_{3}$, leftside $\left(P_{1}, P_{2}, P_{3}\right)$, if $P_{1} \in H P^{-}\left(\overrightarrow{P_{2} P_{3}}\right)$.

The order of the three arguments of the relations rightside and leftside is meaningful: the first argument is the point that is said to be on the rightside (or leftside) of the other two points; the second and third arguments are the points defining a direction that goes from the second point towards the third point. 
It may be observed that three non collinear points are arranged in a triangle. Further, with relations rightside and leftside, it is possible to define an order in the triangles. In particular, if rightside $\left(P_{1}, P_{2}, P_{3}\right)$, then the triangle $\underset{P_{1}}{P_{2}} P_{3}$ is a clockwise ordered triangle. If leftside $\left(P_{1}, P_{2}, P_{3}\right)$, then the triangle $\vec{P}_{1} \stackrel{\Delta}{2}_{2} P_{3}$ is a counter-clockwise ordered triangle (see Fig. 2(b-c)).

In the following, we give the properties of relations rightside and leftside. The first property enables to pass from rightside to leftside and vice versa, while the remaining properties define a cyclic order:

1. $\quad$ rightside $\left(P_{1}, P_{2}, P_{3}\right) \Leftrightarrow$ leftside $\left(P_{1}, P_{3}, P_{2}\right)$;

2. rightside $\left(P_{1}, P_{2}, P_{3}\right) \Rightarrow$ rightside $\left(P_{2}, P_{3}, P_{1}\right)$;

3. rightside $\left(P_{1}, P_{2}, P_{3}\right) \Rightarrow$ rightside $\left(P_{3}, P_{1}, P_{2}\right)$;

4. leftside $\left(P_{1}, P_{2}, P_{3}\right) \Rightarrow$ leftside $\left(P_{2}, P_{3}, P_{1}\right)$;

5. leftside $\left(P_{1}, P_{2}, P_{3}\right) \Rightarrow$ leftside $\left(P_{3}, P_{1}, P_{2}\right)$.

\section{4 "Between" and "Nonbetween" Relations}

The collinear relation can be refined in two relations that are called between and nonbetween. This is possible by assessing whether the first point $P_{1}$ falls inside the segment $\left[P_{2} P_{3}\right]$ or outside it.

Definition 5. A point $P_{1}$ is between two given points $P_{2}$ and $P_{3}$, between $\left(P_{1}, P_{2}, P_{3}\right)$, if one of the following conditions hold:

(a) $P_{1}=P_{2}=P_{3}$;

(b) $P_{1} \in\left[P_{2} P_{3}\right]$ with $P_{2} \neq P_{3}$.

The relation between is illustrated in Fig. 3.

Definition 6. A point $P_{1}$ is nonbetween two given points $P_{2}$ and $P_{3}$, nonbetween $\left(P_{1}, P_{2}, P_{3}\right)$, if collinear $\left(P_{1}, P_{2}, P_{3}\right)$ and:

(a) $P_{1} \notin\left[P_{2} P_{3}\right]$ with $P_{2} \neq P_{3}$;

(b) $P_{1} \neq P_{2}$ with $P_{2}=P_{3}$.

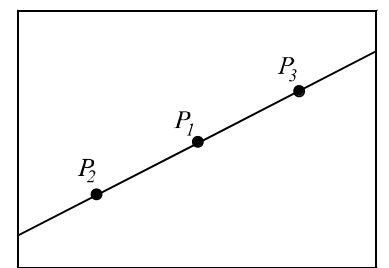

(a)

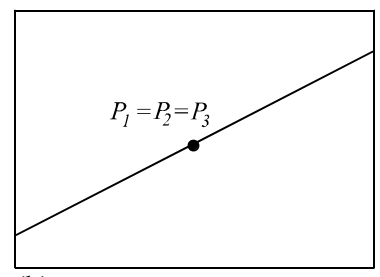

(b)

Fig. 3. The relation between in the general case (a) and in the special case of coincidence of points $P_{2}$ and $P_{3}$ (b) 
The following properties hold. As we can see, if compared to collinear, the relation between is not an equivalence relation anymore, even if some of the properties in the reflexivity, symmetry and transitivity groups are maintained:

1. from the reflexivity group:
a. $\operatorname{between}\left(P_{1}, P_{1}, P_{1}\right)$;
b. nonbetween $\left(P_{1}, P_{2}, P_{2}\right)$;
c. $\operatorname{between}\left(P_{1}, P_{1}, P_{2}\right)$;
d. between $\left(P_{1}, P_{2}, P_{1}\right)$.

2. from the symmetry group:

a. $\quad \operatorname{between}\left(P_{1}, P_{2}, P_{3}\right) \Rightarrow \operatorname{between}\left(P_{1}, P_{3}, P_{2}\right)$;

b. $\quad$ between $\left(P_{1}, P_{2}, P_{3}\right) \Rightarrow$ nonbetween $\left(P_{2}, P_{1}, P_{3}\right)$;

c. $\operatorname{between}\left(P_{1}, P_{2}, P_{3}\right) \Rightarrow$ nonbetween $\left(P_{3}, P_{1}, P_{2}\right)$;

d. nonbetween $\left(P_{1}, P_{2}, P_{3}\right) \Rightarrow$ nonbetween $\left(P_{1}, P_{3}, P_{2}\right)$.

3. transitivity:

a. $\operatorname{between}\left(P_{1}, P_{2}, P_{3}\right) \wedge \operatorname{between}\left(P_{2}, P_{3}, P_{4}\right) \Rightarrow \operatorname{between}\left(P_{1}, P_{3}, P_{4}\right)$;

b. $\operatorname{between}\left(P_{1}, P_{2}, P_{3}\right) \wedge \operatorname{between}\left(P_{2}, P_{3}, P_{4}\right) \Rightarrow \operatorname{between}\left(P_{2}, P_{1}, P_{4}\right)$.

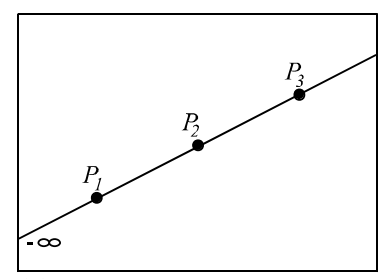

(a)

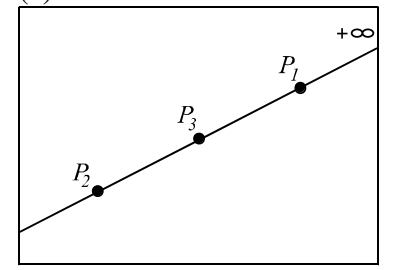

(c)

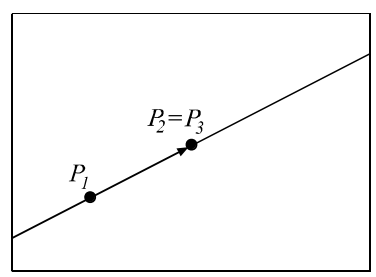

(b)

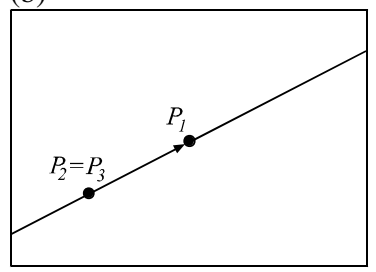

(d)

Fig. 4. The relations before (a-b) and after (c-d)

\section{5 "Before" and "After" Relations}

The nonbetween relation can be refined in the two relations before and after by considering the oriented line $\overrightarrow{P_{2} P_{3}}$ and checking whether the point $P_{1}$ falls inside the interval $\left(-\infty, P_{2}\right)$ or the interval $\left(P_{3},+\infty\right)$, respectively. 
Definition 7. A point $P_{1}$ is before points $P_{2}$ and $P_{3}$, before $\left(P_{1}, P_{2}, P_{3}\right)$, if $\operatorname{collinear}\left(P_{1}, P_{2}, P_{3}\right)$ and:

(a) $P_{1} \in\left(-\infty, P_{2}\right)$, with $P_{2} \neq P_{3}$;

(b) $P_{1} \in \overrightarrow{P_{1} P_{2}}$, with $\left(P_{2}=P_{3}\right) \wedge\left(P_{1} \neq P_{2}\right)$.

Definition 8. A point $P_{1}$ is after points $P_{2}$ and $P_{3}, \operatorname{after}\left(P_{1}, P_{2}, P_{3}\right)$ if $\operatorname{collinear}\left(P_{1}, P_{2}, P_{3}\right)$ and:
(a) $P_{1} \in\left(P_{3},+\infty\right)$, with $P_{2} \neq P_{3}$;
(b) $P_{1} \in \overrightarrow{P_{2} P_{1}}$, with $\left(P_{2}=P_{3}\right) \wedge\left(P_{1} \neq P_{2}\right)$.

In Definitions 7 and 8, part (a) represents the plain case of distinct points $P_{2}$ and $P_{3}$, while part (b) is related to the case of coincident $P_{2}$ and $P_{3}$. For distinct points $P_{2}$ and $P_{3}$ the oriented line $\overrightarrow{P_{2} P_{3}}$ is considered. For coincident $P_{2}$ and $P_{3}$, we consider the line $\overline{P_{1} P_{2}}$ and an arbitrary orientation on this line: if the orientation $\overrightarrow{P_{1} P_{2}}$ is chosen, the relation $\operatorname{before}\left(P_{1}, P_{2}, P_{3}\right)$ holds; all the way round, if the orientation $\overrightarrow{P_{2} P_{1}}$ is chosen, the relation $\operatorname{after}\left(P_{1}, P_{2}, P_{3}\right)$ holds (see Fig. 4).

The properties of before and after relations are the following:

1. before $\left(P_{1}, P_{2}, P_{3}\right) \Rightarrow$ before $\left(P_{3}, P_{2}, P_{1}\right)$;

2. before $\left(P_{1}, P_{2}, P_{3}\right) \Leftrightarrow \operatorname{after}\left(P_{1}, P_{3}, P_{2}\right)$;

3. $\operatorname{before}\left(P_{1}, P_{2}, P_{3}\right) \Leftrightarrow \operatorname{after}\left(P_{3}, P_{1}, P_{2}\right)$;

4. $\quad \operatorname{before}\left(P_{1}, P_{2}, P_{3}\right) \Rightarrow$ between $\left(P_{2}, P_{1}, P_{3}\right)$;

5. $\operatorname{after}\left(P_{1}, P_{2}, P_{3}\right) \Rightarrow \operatorname{after}\left(P_{2}, P_{1}, P_{3}\right)$;

6. $\operatorname{after}\left(P_{1}, P_{2}, P_{3}\right) \Rightarrow \operatorname{between}\left(P_{3}, P_{2}, P_{1}\right)$;

7. $\operatorname{before}\left(P_{1}, P_{2}, P_{3}\right) \wedge$ before $\left(P_{2}, P_{3}, P_{4}\right) \Rightarrow$ before $\left(P_{1}, P_{3}, P_{4}\right)$;

8. $\quad$ before $\left(P_{1}, P_{2}, P_{3}\right) \wedge$ before $\left(P_{2}, P_{3}, P_{4}\right) \Rightarrow$ before $\left(P_{1}, P_{2}, P_{4}\right)$;

9. $\operatorname{after}\left(P_{1}, P_{2}, P_{3}\right) \wedge \operatorname{after}\left(P_{3}, P_{4}, P_{2}\right) \Rightarrow \operatorname{after}\left(P_{1}, P_{4}, P_{2}\right)$;

10. $\operatorname{after}\left(P_{1}, P_{2}, P_{3}\right) \wedge \operatorname{after}\left(P_{3}, P_{4}, P_{2}\right) \Rightarrow \operatorname{after}\left(P_{1}, P_{4}, P_{3}\right)$.

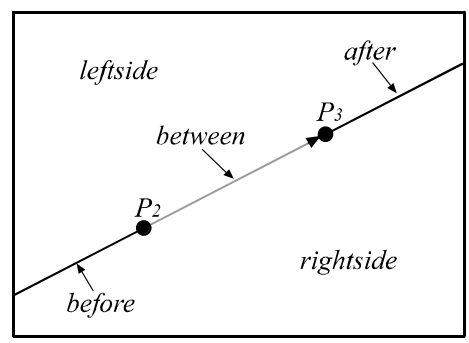

Fig. 5. The five low-level projective relations between points 


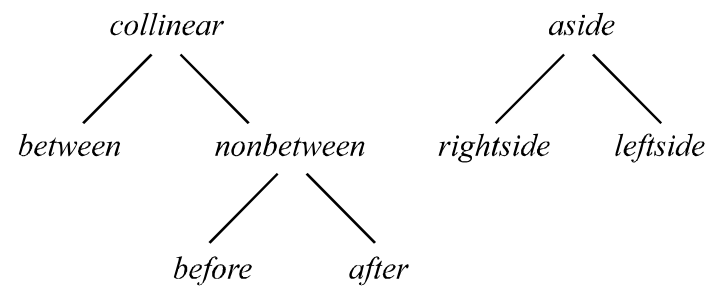

Fig. 6. The hierarchy of projective relations

To summarize, we have built a set of five projective relations between three points of the plane (before, between, after, rightside, leftside) - see Fig. 5. This set is a complete set in the sense that, given any three points, the projective relation between them must be one of the five, and is an independent set of relations, in the sense that, if a given relation between three points is true, then the other four relations must be false. These relations can be hierarchically structured, so to have more general levels of relations: nonbetween is before or after, collinear is between or nonbetween, aside is rightside or leftside (see Fig. 6).

\section{Projective Relations between Regions}

The results of Section 2 were useful to understand the hierarchy of ternary projective relations between three points. Such results immediately followed from the definition of collinearity in a projective space. In this section, we are going to define ternary projective relations between three objects of type region. We will show that it is possible to find plausible definitions for these relations. The definitions for points will be a special case of the definitions for regions. Not all the properties of the relations are maintained passing from points to regions.

\section{1 "Collinear" Relation}

For regions, we will assume the definition given in the OpenGIS Specifications [17], that is, a region is regular closed point set possibly with holes and separate components. For any relation $r(A, B, C)$, the first argument acts as the primary object, while the second and third arguments are reference objects. As a first step, let us introduce the collinear relation between a point and two regions.

Definition 9. A point $P$ is collinear to two regions $B$ and $C, \operatorname{collinear}(P, B, C)$, if there exists a line $l$ intersecting $B$ and $C$ that is also passing trough $P$ :

$$
\exists l,(l \cap B \neq \varnothing) \wedge(l \cap C \neq \varnothing) \mid l \cap P \neq \varnothing .
$$

If the convex hulls of regions $B$ and $C$ are not disjoint, there is always a line passing through $P$ and intersecting $B$ and $C$. Therefore, in this case, we have a degenerate case of collinearity. To avoid it, in the definition of projective relations between three regions, we assume that the regions $B$ and $C$ have disjoint convex hulls. We indicate the convex hull of a region with a unary function $\mathrm{CH}()$. 


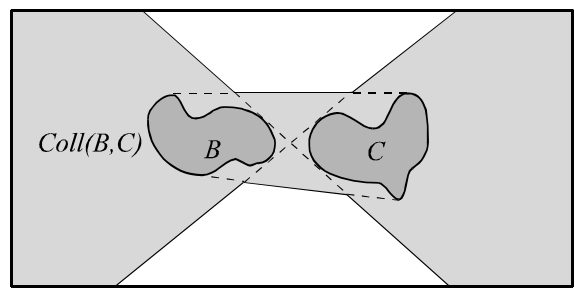

Fig. 7. The part of the plane corresponding to the collinearity region of $B$ and $C$

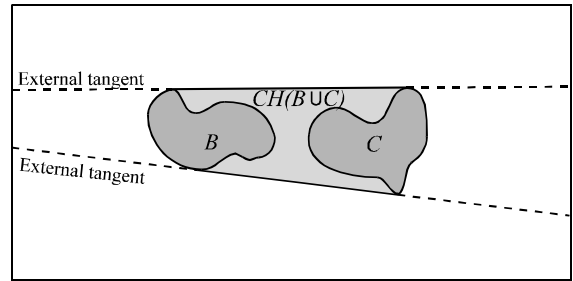

(a)

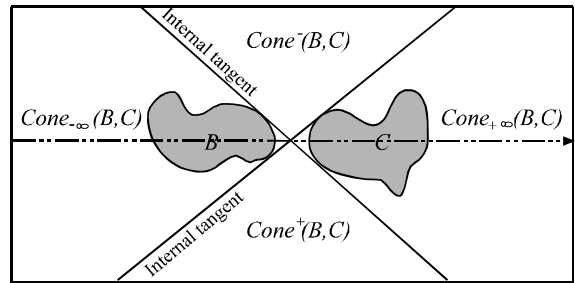

(b)

Fig. 8. The construction to obtain the collinearity region: (a) the common external tangents make up the convex hull of the union of regions $B$ and $C$ and (b) the common internal tangents partition the plane in four cones

Definition 10. Given two regions $B$ and $C$, with $C H(B) \cap C H(C)=\varnothing$, a region $A$ is collinear to regions $B$ and $C$, $\operatorname{colline} \operatorname{ar}(A, B, C)$, if for every point $P \in A$, there exists a line $l$ intersecting $B$ and $C$ that also intersects $P$, that is:

$$
\forall P \in A, \exists l,(l \cap B \neq \varnothing) \wedge(l \cap C \neq \varnothing) \mid l \cap P \neq \varnothing .
$$

For every two regions $B$ and $C$, the relation collinear identifies a part of the plane where a region $A$ completely contained into it is called collinear to $B$ and $C$ (see Fig. 7 ). Let us call this part of the plane the collinearity region of $B$ and $C, \operatorname{Coll}(B, C)$. The collinearity region of $B$ and $C$ can be built by considering all the lines that are intersecting both $B$ and $C$. The boundary of the collinearity region is delimited by four lines that are the common external tangents and the common internal tangents. Common external tangents of $B$ and $C$ are defined by the fact that they also are tangent to the convex hull of the union of $B$ and $C$ (see Fig. 8(a)). Common internal tangents intersect inside the convex hull of the union of regions $B$ and $C$ and divide the plane in four cones (see Fig. 8(b)). In order to distinguish the four cones, we consider an oriented line from region $B$ to region $C$ and we call $C_{-\infty}(B, C)$ the cone that contains region $B$, Cone $_{+\infty}(B, C)$ the cone that contains region $C$, $\operatorname{Cone}^{+}(B, C)$ the cone that is to the right of the oriented line, Cone ${ }^{-}(B, C)$ the cone that is to the left of the oriented line. In terms of these subregions, the collinearity region is equivalent to the following:

$$
\operatorname{Coll}(B, C)=\text { Cone }_{-\infty}(B, C) \cup \text { Cone }_{+\infty}(B, C) \cup C H(B \cup C) .
$$

We showed in Section 2 that the relation collinear among points is a ternary equivalence relation. The relation collinear for regions is not an equivalence relation because we lose transitivity. The only remaining properties are reflexivity and symmetry: 
1. The collinear relation is reflexive:
a. collinear $(A, A, A)$;
b. collinear $(A, B, B)$;
c. collinear $(A, A, B)$;
d. collinear $(A, B, A)$.

2. The collinear relation is symmetric:
a. collinear $(A, B, C) \Rightarrow$ collinear $(A, C, B)$;
b. collinear $(A, B, C) \Rightarrow$ collinear $(B, A, C)$;
c. collinear $(A, B, C) \Rightarrow$ collinear $(C, A, B)$.

When discussing properties of relations, the role of primary object and the reference objects are often exchanged: therefore, to avoid degenerate cases of collinearity, we must assume that the two reference objects have disjoint convex hulls.

\section{2 "Aside" Relation}

If a region $A$ is collinear to regions $B$ and $C$, it means that it is entirely contained in the collinearity region of $B$ and $C$. If the relation aside is true, it means that region $A$ is entirely contained in the complement of the collinearity region. We will see at the end of this section how the model takes into account configurations where region $A$ is partly inside and partly outside the collinearity region.

Definition 11. A region $A$ is aside two regions $B$ and $C$, aside $(A, B, C)$, if there is no line $l$ intersecting $B$ and $C$ that also intersects $A$ :

$$
\forall l,(l \cap B \neq \varnothing) \wedge(l \cap C \neq \varnothing) \Rightarrow l \cap A=\varnothing .
$$

The aside relation is symmetric:

$$
\begin{aligned}
& \operatorname{aside}(A, B, C) \Rightarrow \operatorname{aside}(A, C, B) ; \\
& \operatorname{aside}(A, B, C) \Rightarrow \operatorname{aside}(B, A, C) ; \\
& \operatorname{aside}(A, B, C) \Rightarrow \operatorname{aside}(C, A, B) .
\end{aligned}
$$

\section{3 "Rightside" and "Leftside" Relation}

The rightside and leftside relations are refinements of the aside relation, which are obtained by considering a region $A$ that falls inside the two cones $\operatorname{Cone}^{+}(B, C)$ or Cone $^{-}(B, C)$, respectively.

Definition 12. A region $A$ is rightside of two regions $B$ and $C$, rightside $(A, B, C)$, if $A$ is contained inside $\operatorname{Cone}^{+}(B, C)$ minus the convex hull of the union of regions $B$ and $C$, that is, if $A \subset\left(\right.$ Cone $\left.^{+}(B, C)-C H(B \cup C)\right)$. 
Definition 13. A region $A$ is leftside of two regions $B$ and $C$, leftside $(A, B, C)$, if $A$ is contained inside $C_{0 n e}^{-}(B, C)$ minus the convex hull of the union of regions $B$ and $C$, that is, if $A \subset\left(\right.$ Cone $\left.^{-}(B, C)-C H(B \cup C)\right)$.

The following are the properties of relations leftside and rightside for regions. With respect to the corresponding properties we had for points, there is no more a strict cyclic order but a more permissive form of it:

1. rightside $(A, B, C) \Leftrightarrow$ leftside $(A, C, B)$;

2. rightside $(A, B, C) \Rightarrow$ rightside $(B, C, A) \vee(\operatorname{rightside}(B, C, A) \wedge \operatorname{between}(B, C, A))$;

3. rightside $(A, B, C) \Rightarrow$ rightside $(C, A, B) \vee(\operatorname{rightside}(C, A, B) \wedge$ between $(C, A, B))$;

4. leftside $(A, B, C) \Rightarrow$ leftside $(B, C, A) \vee(\operatorname{leftside}(B, C, A) \wedge \operatorname{between}(B, C, A))$;

5. leftside $(A, B, C) \Rightarrow$ leftside $(B, C, A) \vee(\operatorname{leftside}(B, C, A) \wedge \operatorname{between}(B, C, A))$.

\section{4 "Between" and "Nonbetween" Relations}

The between and nonbetween relations are a refinement of the collinear relation. If region A falls inside the convex hull of the union of regions $B$ and $C$, then by definition it is between $B$ and $C$, otherwise is nonbetween.

Definition 14. A region $A$ is between two regions $B$ and $C$, between $(A, B, C)$, if $A \subseteq C H(B \cup C)$

Definition 15. A region $A$ is nonbetween two regions $B$ and $C$, nonbetween $(A, B, C)$, if $A \subset\left(\right.$ Cone $_{-\infty}(B, C) \cup$ Cone $\left._{+\infty}(B, C)\right)-C H(B \cup C)$.

As we did for points, the following are the properties from the reflexive, symmetric and transitive groups that are maintained for the relation between among regions:

1. from the reflexivity group:
a. between $(A, A, A)$;
b. between $(A, A, B)$;
c. $\operatorname{between}(A, B, A)$.

2. from the symmetry group:

a. between $(A, B, C) \Rightarrow$ between $(A, C, B)$;

b. between $(A, B, C) \Rightarrow$ nonbetween $(B, A, C)$;

c. between $(A, B, C) \Rightarrow$ nonbetween $(C, A, B)$;

d. nonbetween $(A, B, C) \Rightarrow$ nonbetween $(A, C, B)$.

3. transitivity:

$\operatorname{between}(A, B, C) \wedge \operatorname{between}(B, C, D) \Rightarrow \operatorname{between}(A, C, D)$.

\section{5 "Before" and "After" Relations}

The relation nonbetween can be refined by checking whether region $A$ falls inside Cone $_{-\infty}(B, C)$ or Cone $e_{+\infty}(B, C)$, obtaining the relations before and after, respectively. 
Definition 16. A region $A$ is before two regions $B$ and $C$, before $(A, B, C)$, if $A \subset$ Cone $_{-\infty}(B, C)-C H(B \cup C)$.

Definition 17. A region $A$ is after two regions $B$ and $C$, after $(A, B, C)$, if $A \subset$ Cone $_{+\infty}(B, C)-C H(B \cup C)$.

We can identify the following properties for the relations before and after among regions:

1. before $(A, B, C) \Rightarrow$ before $(C, B, A) \vee($ before $(C, B, A) \wedge$ aside $(C, B, A))$;

2. before $(A, B, C) \Leftrightarrow \operatorname{after}(A, C, B)$;

3. before $(A, B, C) \Rightarrow \operatorname{after}(C, A, B) \vee(\operatorname{after}(C, A, B) \wedge \operatorname{aside}(C, A, B))$;

4. $\operatorname{before}(A, B, C) \Rightarrow \operatorname{between}(B, A, C) \vee($ between $(B, A, C) \wedge$ aside $(B, A, C))$;

5. $\operatorname{after}(A, B, C) \Rightarrow \operatorname{after}(B, A, C) \vee(\operatorname{after}(B, A, C) \wedge \operatorname{aside}(B, A, C))$;

6. $\operatorname{after}(A, B, C) \Rightarrow$ before $(B, C, A) \vee($ before $(B, C, A) \wedge \operatorname{aside}(B, C, A))$;

7. $\operatorname{after}(A, B, C) \Rightarrow$ between $(C, B, A) \vee($ between $(C, B, A) \wedge$ aside $(C, B, A))$;

8. before $(A, B, C) \wedge$ before $(B, C, D) \Rightarrow$ before $(A, C, D) \vee($ before $(A, C, D) \wedge$ aside $(A, C, D)) \vee$ aside $(A, C, D)$;

9. $\operatorname{before}(A, B, C) \wedge$ before $(B, C, D) \Rightarrow$

before $(A, B, D) \vee($ before $(A, B, D) \wedge$ aside $(A, B, D)) \vee$ aside $(A, B, D)$;

10. $\operatorname{after}(A, B, C) \wedge \operatorname{after}(C, D, B) \Rightarrow$

$\operatorname{after}(A, D, B) \vee(\operatorname{after}(A, D, B) \wedge$ aside $(A, D, B)) \vee$ aside $(A, D, B)$.

11. $\operatorname{after}(A, B, C) \wedge \operatorname{after}(C, D, B) \Rightarrow$

$\operatorname{after}(A, D, C) \vee(\operatorname{after}(A, D, C) \wedge$ aside $(A, D, C)) \vee \operatorname{aside}(A, D, C)$.

The set of five projective relations before, between, after, rightside, and leftside can be used as a set of basic relations to build a model for all projective relations between three regions of the plane. For the sake of simplicity, let us give a name to the regions of the plane corresponding to the basic relations (see also Fig. 9):

Before $(B, C)=$ Cone $_{-\infty}(B, C)-C H(B \cup C)$;

$\operatorname{After}(B, C)=$ Cone $_{+\infty}(B, C)-C H(B \cup C)$;

Rightside $(B, C)=$ Cone $^{+}(B, C)-C H(B \cup C)$;

Leftside $(B, C)=$ Cone $^{-}(B, C)-C H(B \cup C)$;

Between $(B, C)=C H(B \cup C)$.

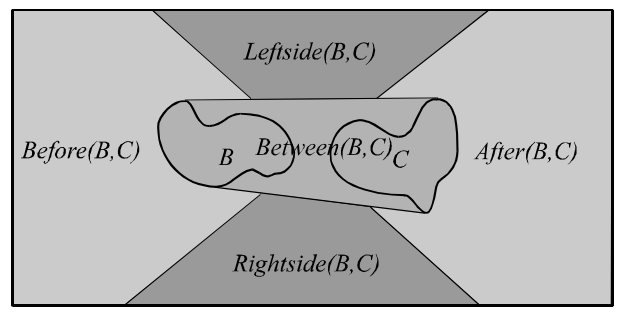

Fig. 9. The partition of the plane in five regions 
The model, that we call the 5-intersection, is synthetically expressed by a matrix of five values that are the empty/non-empty intersections of a region $A$ with the five regions defined above:

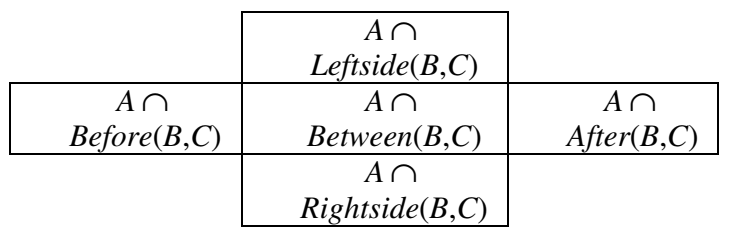

In the matrix, a value 0 indicates an empty intersection, while a value 1 indicates a non-empty intersection. The five basic relations correspond to values of the matrix with only one non-empty value:

$$
\begin{aligned}
& \text { before }(A, B, C):\left(\begin{array}{lll}
0 & \\
1 & 0 & 0 \\
0
\end{array}\right) ; \operatorname{between}(A, B, C):\left(\begin{array}{lll}
0 & \\
0 & 1 & 0 \\
0 & 0
\end{array}\right) ; \operatorname{after}(A, B, C):\left(\begin{array}{lll}
0 & \\
0 & 0 & 1 \\
0 &
\end{array}\right) ; \\
& \text { rightside }(A, B, C):\left(\begin{array}{lll}
0 & 0 \\
0 & 0 & 0 \\
& 1
\end{array}\right) ; \quad \operatorname{leftside}(A, B, C):\left(\begin{array}{lll}
1 & 1 \\
0 & 0 & 0 \\
0
\end{array}\right) .
\end{aligned}
$$

In total, the 5-intersection matrix can have $2^{5}$ different values that correspond to the same theoretical number of projective relations. Excluding the configuration with all zero values which cannot exist, we are left with 31 different projective relations between the three regions $A, B$ and $C$. In Fig. 10-13, we have some examples of the 31 projective relations. Previously defined nonbetween, collinear and aside relations can be expressed in terms of the 5-intersection as follows:

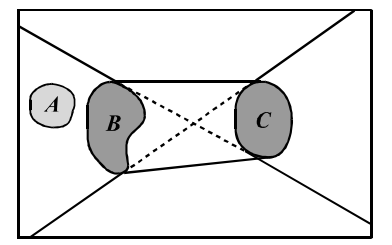

$\left(\begin{array}{lll} & 0 & \\ 1 & 0 & 0 \\ & 0 & \end{array}\right)$

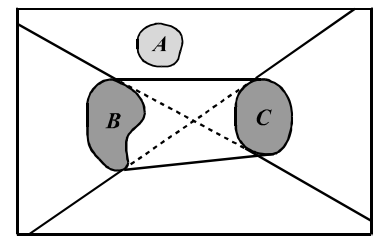

$\left(\begin{array}{lll} & 1 & \\ 0 & 0 & 0 \\ & 0 & \end{array}\right)$
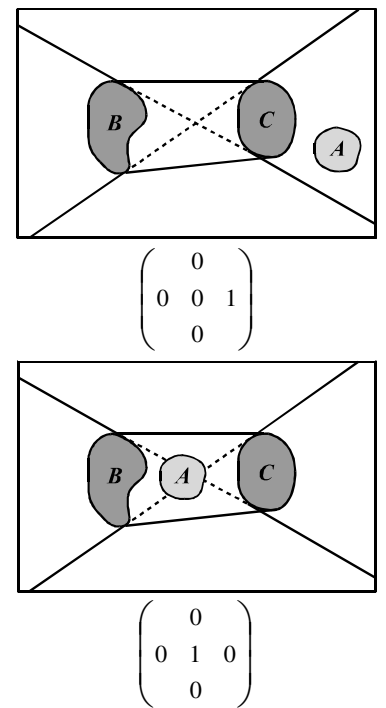

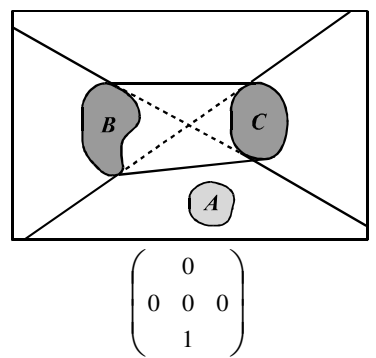

Fig. 10. The projective relations with object $A$ intersecting only one of the regions of the plane 


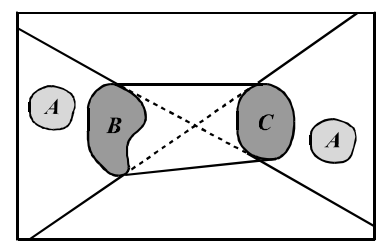

$\left(\begin{array}{lll} & 0 & \\ 1 & 0 & 1 \\ & 0 & \end{array}\right)$

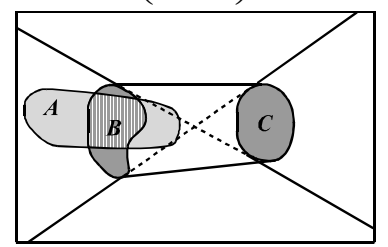

$\left(\begin{array}{lll} & 0 & \\ 1 & 1 & 0 \\ & 0 & \end{array}\right)$

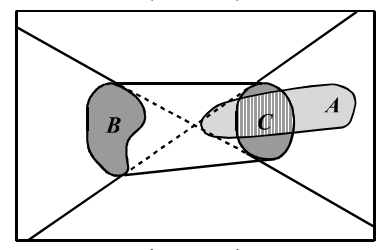

$\left(\begin{array}{lll} & 0 & \\ 0 & 1 & 1 \\ & 0 & \end{array}\right)$

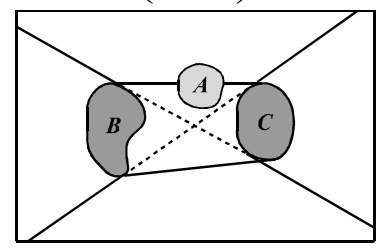

$\left(\begin{array}{lll} & 1 & \\ 0 & 1 & 0 \\ & 0 & \end{array}\right)$

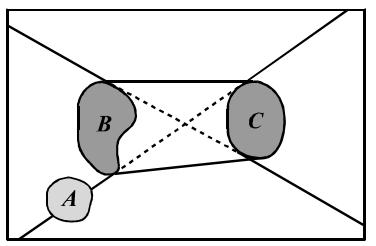

$\left(\begin{array}{lll} & 0 & \\ 1 & 0 & 0 \\ & 1 & \end{array}\right)$

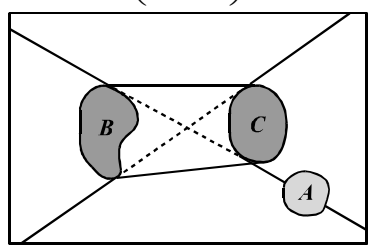

$\left(\begin{array}{lll} & 0 & \\ 0 & 0 & 1 \\ & 1 & \end{array}\right)$

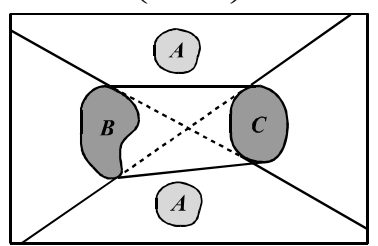

$\left(\begin{array}{lll} & 1 & \\ 0 & 0 & 0 \\ & 1 & \end{array}\right)$

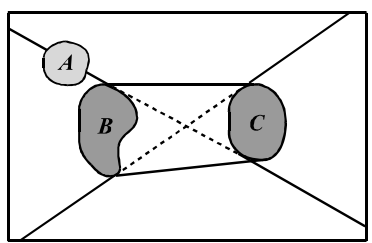

$\left(\begin{array}{lll} & 1 & \\ 1 & 0 & 0 \\ & 0\end{array}\right)$

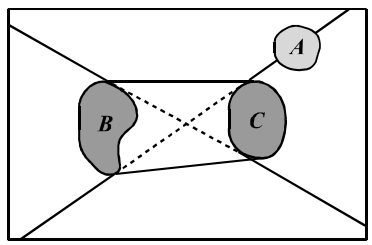

$\left.\begin{array}{lll} & 1 & \\ 0 & 0 & 1 \\ & 0 & \end{array}\right)$

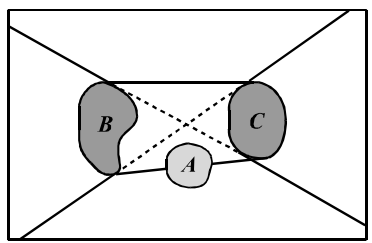

$\left(\begin{array}{lll} & 0 & \\ 0 & 1 & 0 \\ & 1 & \end{array}\right)$

Fig. 11. The projective relations with object $A$ intersecting two regions of the plane

nonbetween $(A, B, C): \quad\left(\begin{array}{lll}0 & \\ 1 & 0 & 0 \\ 0 & 0\end{array}\right) \vee\left(\begin{array}{lll} & 0 \\ 0 & 0 & 1 \\ 0\end{array}\right) \vee\left(\begin{array}{lll}0 & \\ 1 & 0 & 1 \\ 0 & 0\end{array}\right)$; collinear $(A, B, C):\left(\begin{array}{lll}0 & \\ 1 & 0 & 0 \\ 0 & 0\end{array}\right) \vee\left(\begin{array}{lll} & 0 \\ 0 & 1 & 0 \\ 0\end{array}\right) \vee\left(\begin{array}{lll} & 0 & \\ 0 & 0 & 1 \\ 0 & 0\end{array}\right) \vee\left(\begin{array}{lll} & 0 \\ 1 & 1 & 0 \\ 0 & 0\end{array}\right) \vee$ $\left(\begin{array}{lll}0 & \\ 1 & 0 & 1 \\ 0 & 0\end{array}\right) \vee\left(\begin{array}{lll}0 & \\ 0 & 1 & 1 \\ 0 & 1\end{array}\right) \vee\left(\begin{array}{lll}0 & \\ 1 & 1 & 1 \\ 0 & 1\end{array}\right)$ 


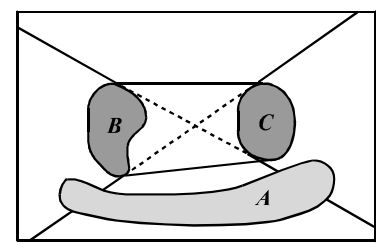

$\left(\begin{array}{lll} & 0 & \\ 1 & 0 & 1 \\ & 1 & \end{array}\right)$

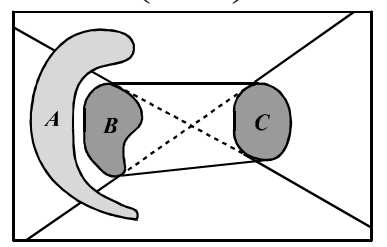

$\left(\begin{array}{lll} & 1 & \\ 1 & 0 & 0 \\ & 1 & \end{array}\right)$

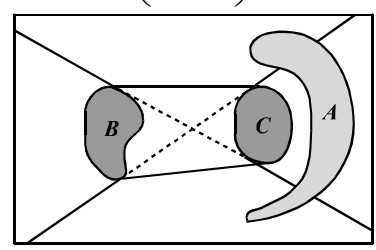

$\left(\begin{array}{lll} & 1 & \\ 0 & 0 & 1 \\ & 1 & \end{array}\right)$

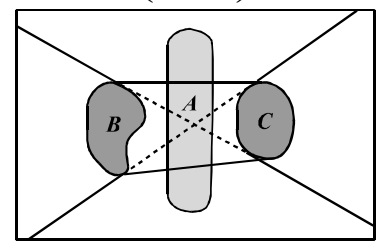

$\left(\begin{array}{lll} & 1 & \\ 0 & 1 & 0 \\ & 1\end{array}\right)$

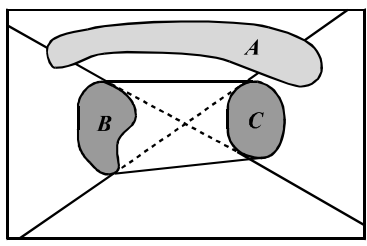

$\left(\begin{array}{lll} & 1 & \\ 1 & 0 & 1 \\ & 0 & \end{array}\right)$

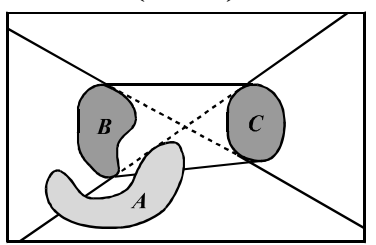

$\left(\begin{array}{lll} & 0 & \\ 1 & 1 & 0 \\ & 1 & \end{array}\right)$

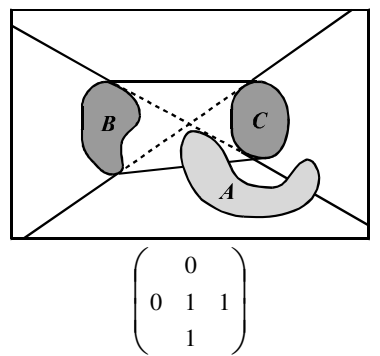

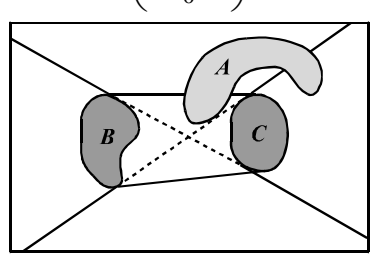

$\left(\begin{array}{lll} & 1 & \\ 0 & 1 & 1 \\ & 0 & \end{array}\right)$

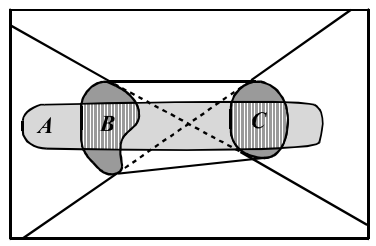

$\left(\begin{array}{lll}1 & 1 & 1 \\ & 0 & \end{array}\right)$

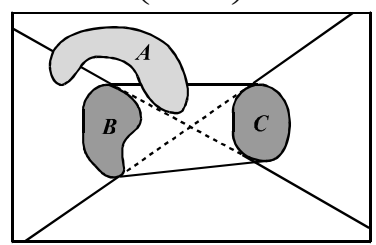

$\left(\begin{array}{lll} & 1 & \\ 1 & 1 & 0 \\ & 0 & \end{array}\right)$

Fig. 12. The projective relations with object $A$ intersecting three regions of the plane

$$
\operatorname{aside}(A, B, C):\left(\begin{array}{lll} 
& 0 \\
0 & 0 & 0 \\
1
\end{array}\right) \vee\left(\begin{array}{lll}
1 & 1 \\
0 & 0 & 0 \\
0 & 0
\end{array}\right) \vee\left(\begin{array}{lll} 
& 1 & \\
0 & 0 & 0 \\
& 1
\end{array}\right) .
$$

Besides the relations above, it is possible to assign a name to other relations by using a combination of the basic relation names, for example, the following relation would be a "before and rightside":

$$
\left(\begin{array}{lll} 
& 0 & \\
1 & 0 & 0 \\
& 1
\end{array}\right)
$$



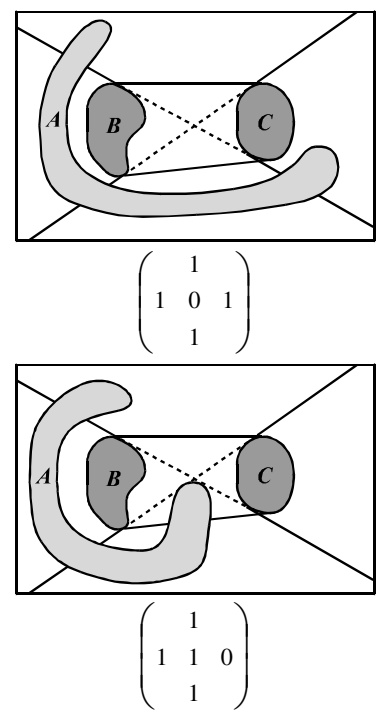
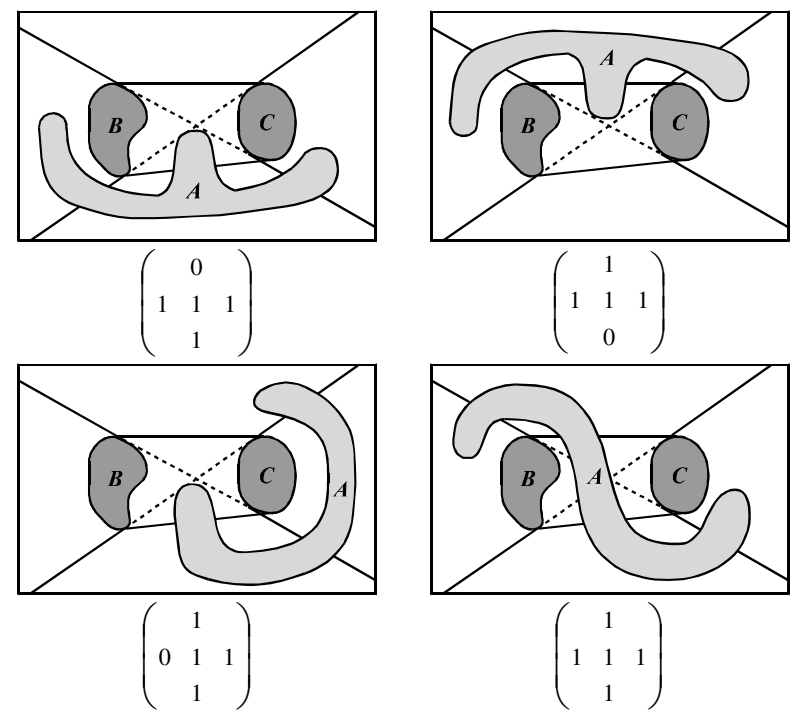

Fig. 13. The projective relations with object $A$ intersecting four or five regions of the plane

\section{Further Research}

This paper introduces a set of jointly exhaustive and pairwise disjoint projective relations between three regions of the plane. The number of relations is 31 and is obtained by considering the collinearity of three points as the basic projective invariant. The strengths of this model are the independence from any external frame of reference and the capacity of taking the actual size and shape of objects into account for defining the relations.

The model presented in this paper is the first step of future research on projective relations. The proofs of the formal properties of relations will be given. The relations for lines and also mixed cases (region/point, region/line, line/point) will be developed as well as a $3 \mathrm{D}$ extension of the model. The reasoning system will be improved to have rules for symmetry and transitivity by means of composition tables: with regard to symmetric properties, given the projective relation $r(A, B, C)$, the rules will give $r(A, C, B), r(B, A, C)$ and $r(C, A, B)$; with regard to transitive properties, given the relations $r(A, B, C)$ and $r(B, C, D)$, the rules will give $r(A, C, D)$.

Furthermore, the relations will be extended for scenes of more than three objects: as a combination of ternary relations, it will be possible to express relations such as "surrounded by" or "in the middle of". Another step will be the evaluation of algorithms to find out the partition of the plane in five regions for any pair of reference objects and the implementation of projective relations in a spatial database system.

Acknowledgements. This work was supported by M.I.U.R. under project "Representation and management of spatial and geographic data on the Web". 


\section{References}

1. Clementini, E. and P. Di Felice, Spatial Operators. ACM SIGMOD Record, 2000. 29(3): p. 31-38.

2. Clementini, E., P. Di Felice, and D. Hernández, Qualitative representation of positional information. Artificial Intelligence, 1997. 95: p. 317-356.

3. Clementini, E., P. Di Felice, and P. van Oosterom, A Small Set of Formal Topological Relationships Suitable for End-User Interaction, in Advances in Spatial Databases - Third International Symposium, SSD '93, D. Abel and B.C. Ooi, Editors. 1993, Springer-Verlag: Berlin. p. 277-295.

4. Dugat, V., P. Gambarotto, and Y. Larvor. Qualitative Theory of Shape and Orientation. in Proc. of the 16th Int. Joint Conference on Artificial Intelligence (IJCAI'99). 1999. Stockolm, Sweden: Morgan Kaufmann Publishers. p. 45-53.

5. Egenhofer, M.J., Deriving the composition of binary topological relations. Journal of Visual Languages and Computing, 1994. 5(1): p. 133-149.

6. Egenhofer, M.J. and J.R. Herring, Categorizing Binary Topological Relationships Between Regions, Lines, and Points in Geographic Databases. 1991, Department of Surveying Engineering, University of Maine, Orono, ME.

7. Freksa, C., Using Orientation Information for Qualitative Spatial Reasoning, in Theories and Models of Spatio-Temporal Reasoning in Geographic Space, A.U. Frank, I. Campari, and U. Formentini, Editors. 1992, Springer-Verlag: Berlin. p. 162-178.

8. Gapp, K.-P. Angle, Distance, Shape, and their Relationship to Projective Relations. in Proceedings of the 17th Conference of the Cognitive Science Society. 1995. Pittsburgh, PA.

9. Gapp, K.-P. From Vision to Language: A Cognitive Approach to the Computation of Spatial Relations in 3D Space. in Proc. of the First European Conference on Cognitive Science in Industry. 1994. Luxembourg. p. 339-357.

10. Goyal, R. and M.J. Egenhofer, Cardinal directions between extended spatial objects. IEEE Transactions on Knowledge and Data Engineering, 2003. (in press).

11. Hernández, D., Qualitative Representation of Spatial Knowledge. Lecture Notes in Artificial Intelligence. Vol. LNAI 804. 1994, Berlin: Springer-Verlag.

12. Isli, A. Combining Cardinal Direction Relations and other Orientation Relations in QSR. in AI\&M 14-2004, Eighth International Symposium on Artificial Intelligence and Mathematics. January 4-6, 2004. Fort Lauderdale, Florida.

13. Kray, C. and A. Blocher. Modeling the Basic Meanings of Path Relations. in Proc. of the 16th International Joint Conference on Artificial Intelligence (IJCAI-99). 1999. Stockolm, Sweden: Morgan Kaufmann Publishers. p. 384-389.

14. Kulik, L., et al. A graded approach to directions between extended objects. in Proc. of the 2nd Int. Conf. on Geographic Information Science. 2002. Boulder, CO: Springer. p. 119131.

15. Kulik, L. and A. Klippel, Reasoning about Cardinal Directions Using Grids as Qualitative Geographic Coordinates, in Spatial Information Theory. Cognitive and Computational Foundations of Geographic Information Science: International Conference COSIT'99, C. Freksa and D.M. Mark, Editors. 1999, Springer. p. 205-220.

16. Moratz, R. and K. Fischer. Cognitively Adequate Modelling of Spatial Reference in Human-Robot Interaction. in Proc. of the 12th IEEE International Conference on Tools with Artificial Intelligence, ICTAI 2000. 2000. Vancouver, BC, Canada. p. 222-228.

17. OpenGIS Consortium, OpenGIS Simple Features Specification for SQL. 1998.

18. Retz-Schmidt, G., Various Views on Spatial Prepositions. AI Magazine, 1988. 9(2): p. 95105.

19. Schlieder, C., Reasoning about ordering, in Spatial Information Theory: A Theoretical Basis for GIS - International Conference, COSIT'95, A.U. Frank and W. Kuhn, Editors. 1995, Springer-Verlag: Berlin. p. 341-349. 
20. Schmidtke, H.R., The house is north of the river: Relative localization of extended objects, in Spatial Information Theory. Foundations of Geographic Information Science: International Conference, COSIT 2001, D.R. Montello, Editor. 2001, Springer. p. 415-430.

21. Scivos, A. and B. Nebel, Double-Crossing: Decidability and Computational Complexity of a Qualitative Calculus for Navigation, in Spatial Information Theory. Foundations of Geographic Information Science: International Conference, COSIT 2001, D.R. Montello, Editor. 2001, Springer. p. 431-446.

22. Struik, D.J., Projective Geometry. 1953, London: Addison-Wesley.

23. Vorwerg, C., et al. Projective relations for 3D space: Computational model, application, and psychological evaluation. in Proc. of the 14th National Conference on Artificial Intelligence and 9th Innovative Applications of Artificial Intelligence Conference, AAAI 97, IAAI 97. 1997. Providence, Rhode Island: AAAI Press / The MIT Press. p. 159-164.

24. Waller, D., et al., Place learning in humans: The role of distance and direction information. Spatial Cognition and Computation, 2000. 2: p. 333-354. 